\title{
Nanotechnology Can be Helped by a New Technology for Electromagnetic Waves
}

\author{
J. M. Velázquez-Arcos \\ Departamento de Ciencias Básicas, Universidad Autónoma Metropolitana, México D.F., 02200, México
}

\begin{abstract}
On this paper we propose the use of a recently developed technology about the substitution of metamaterials with electro magnetic time reversal procedures for apply in some issues of nanotechnology. The general concept is to take advantage of two important facts: first we can localize the electro magnetic signals much better; secondly, in many cases we can use waves of minor frequency than that we would use in absence of this technology. To this end we give some examples of specific research where it is possible to apply the vector-matrix formalis $m$ to nanotechnology.
\end{abstract}

Keywords Nanotechnology, Time Reversal, Subwavelength Focusing

\section{Introduction}

Nowadays we have an intense research about several new fields of application of nanotechnology; since development of materials with extraordinary physical properties like the very strong carbon nanotubes, to the modeling of artificial atoms that interact with single mic rowave photons that could be push the quantum computers near to reality. Many of those fields are close related to the emp loy of microwaves in a natural way because of possibility to an easy manipulation of this range of the electromagnetic spectrum in addition to their propert ies to carry information on communicat ions. But one of the tools employed to magnify images and focus signals is the use of metamaterials. Metamaterials are also a new exciting field of physics research and have his own essential or enclosure applications. Nevertheless, metamaterials are not the only possibility of access to the nanotechnology world. Thanks to many recent works we know that there are other possibilities like the use of time reversal methods to reproduce and even improve the results of meta materials in a large class of nanotechnology problems. The question is then when we can use alternative time reversal methods?

The answer is in the manufacture of metamaterials, because usually it is a difficult and expensive task. So, it is justified in many cases to employ time reversal methods.

In this work, we give in section 3 a blueprint of a general method we call the vector-matrix formalis $m$ that gives us an easy to apply way to overco me the diffraction limit and focus a microwave signal. As a preface we before comment in section 2 some recent researches on nanotechnology and

* Corresponding author:

jmva@correo.azc.uam.mx (J. M. Velázquez-Arcos)

Published online at http://journal.sapub.org/nn

Copyright (C) 2012 Scientific \& Academic Publishing. All Rights Reserved finally in section 4 we propose how we could improve the results obtained by using time reversal procedures.

The novelty of this work is in the tool itself, but also in the blueprint of its applications to nanotechnology, because we can now design (By building the kernel (3)) devices that will can focus las er or micro waves beams with a minor frequency that can be focused more precisely. To be more specific, we show with an example for electromagnetic pincers, how the magnetic moment of the target reacts with the electromagnetic field of the beams; or in the example of artific ial ato ms how a better electro magnetic localization of a microwave beam could improve the performance of a new kind of Nano-devices.

\section{A sight to Nanotechnology}

In a very interesting paper of 2007[1], the authors have find that microwaves shows his particle identity on a class of macroscopic systems they called artificial ato ms (see figure 1). So they observed the effects of quantum mechanics but in a scale near to a visib le one. These results marked a clear line of res earch about the manufacture of quantumcomputers and other related devices[2 and 3] that now seems to be near to reality.

The process they observed was the interaction of microwave photons with the artificial atoms which have a size very much larger than the traditional quantum doma in.

Direct implications are between others, that communication process on Nano-devices can be optimized by allow the long-range neighbor interactions without errors.

Because the microwave interaction also can reach atomic spins, molecules and even atoms, other nanotechnology fields can be grows, for example manipulation of nanoparticles by using the interaction between particle spins and electromagnetic beams $[4,5$, and 6] which permits the 
building of electro magnetic pincers .

Maybe the more known field is the development of optical amplifiers or lenses which provides well defined images of the Nano-scale world. The general mechanism is the recovery of the so named near field that consist in evanescent waves[7]. This is a field where the use of metamaterials is strongly used (see figure 2) and the wavelength is less restricted to micro waves, that is the images usually are in the visible spectrum. One of these simplest devices consists in a thin layer of a semiconductor with a silver layer as a top.

The manufacturing of Nano-engines is another very exciting branch of nanotechnology with amazing results which includes not only engines but also Nano-structures that seems to be automobiles which can move by its own resources and whose individual parts are made with single molecules. Even when there is not a necessary relation with this singular engines with microwaves it is clear that his composition are inclined to react with this range of wavelengths (see for example[8] ). Nano-medicine is also one of the more active branches of Nano-technology research with an obvious great importance whose real devices depend on microwave communications between the device and the medical observer.

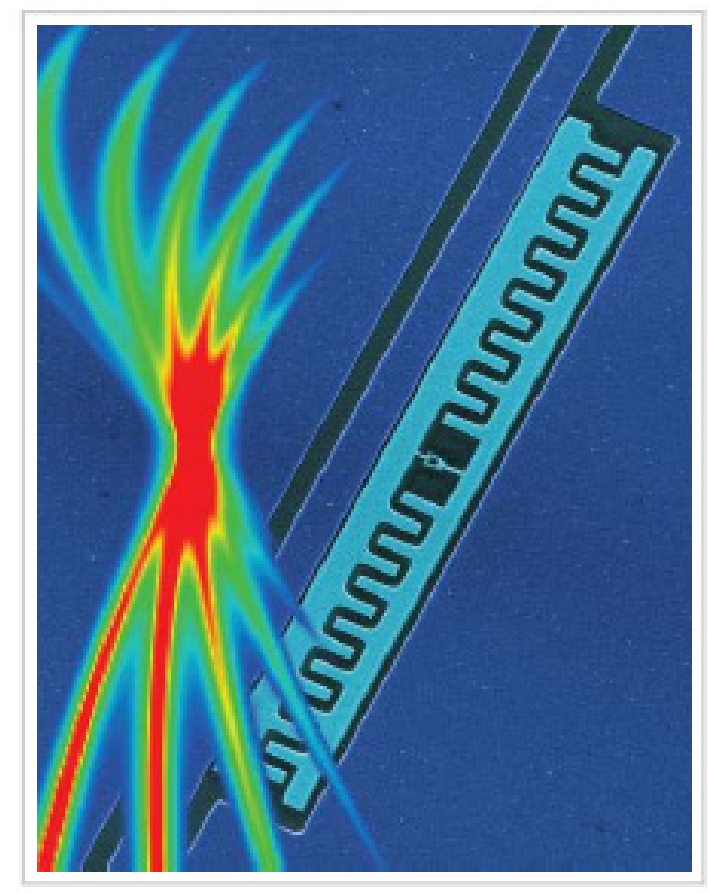

Figure 1. A sample line graph using colours which contrast well both on screen and on a black-and-white hard copy[1]

\section{Why Time Reversal and Not Meta-materials?}

On section 2 we have underline the close relation between Nanotechnology and the mic rowaves used at different levels, not only as a direct reactive agent but also as a source of power and a carrier of information. Also, we commented that the use of metamaterials is very frequently in order to localize electro magnetic signals. But in many cases, the sole procedure to build metamaterials is difficult and expensive. A relatively recent tool has been developed that allow to make an extremely efficient signal localization $(\sim \lambda / 30)$ for microwaves about $2.5 \mathrm{GHz}[4]$ and it is a fact that is superior to the more nearest results with the use of metamaterials $(\sim \lambda / 14)$. This tool is known as Time Reversal (TR) and consists in sending a signal but in time reversal order. This procedure was developed first to localize acoustic signals, but then the researchers export the techniques to electromagnetic waves. But how a TR can recover the evanescent waves like a meta material? In 2009[9] we discussed that the Green's function needed for TR (for acoustic signals) have similar properties than the explicit expressions of complex index of refraction in a metamaterial (for electromagnetic waves), then it was a natural feeling to hope that must exist a formalism that can reproduce the effects of a metamaterial under the electro magnetic field but without his physical existence (of the metamaterials). But experiments support this hope as we have mentioned above, so we write a vector-matrix formalis $\mathrm{m}[10]$ that contains all the information about an specific discrete electromagnetic system and that can be applied to improve the process of sending a signal and localize them with the minor loss of information. Then we present the formalism as the final vector-matrix equation and his possible applications.

Before this we recognize some physical limits. First, only discrete systems can be considered; secondly we suppose that the position of the emitters-receptors is chosen in a non-periodic array and third, some of the sink terms (at least one) must be located in the near zone. The three conditions guarantee to avoid that the negative effects of interference destroy information and that we preserve a mathematical base to represent the complete electro magnetic process. The vector-matrix equation for a TR is:

For row vectors

$$
\overline{\mathrm{f}}^{m}(\omega)=\overline{\mathrm{f}}^{n(\circ)}(\omega)\left[\mathbf{1}+\mathbf{R}^{*}(\omega)\right]_{m}^{n}
$$

And for column vectors

$$
\bar{f}^{n}(\omega)=[\mathbf{1}+\mathbf{R}(\omega)]_{m}^{n} \bar{f}^{m(\circ)}(\omega)
$$

Where the kernel is defined as

$$
\mathbf{R}_{j, k, n}^{m^{*}}(\omega)=\left\{\begin{array}{c}
0 \quad \text { if } \quad j=k \\
A_{j}^{m, n^{*}} \mathcal{G}_{\omega}^{m, n^{*}}\left(\bar{r}_{k}, \bar{r}_{j}\right) \text { if } \quad j \neq k
\end{array}\right.
$$

Here

$$
A_{j}^{m, n^{*}}
$$

Is the interaction

$$
\mathcal{G}_{\omega}^{m, n^{*}}\left(\bar{r}_{k}, \bar{r}_{j}\right)
$$

It is the Fourier transform of the complete Green's function.

The electromagnetic field vectors are

$$
\begin{aligned}
& \overline{\mathrm{f}}^{m}(\omega) \text { row vectors } \\
& \bar{f}^{n}(\omega)_{\text {column vectors }}
\end{aligned}
$$


The source-sink terms are

$$
\begin{aligned}
& \overline{\mathrm{f}}^{n(\circ)}(\omega) \text { row vectors } \\
& \bar{f}^{m(\circ)}(\omega)_{\text {column vectors }}
\end{aligned}
$$

The notation on (1) (or (2) for column vectors) can be explained and illustrated with the case with three emitters and three receptors:

First we build the row "vector"

$$
\bar{f}^{n}=\left(\mathrm{f}_{1}^{n}, \mathrm{f}_{2}^{n}, \mathrm{f}_{3}^{n}\right)
$$

And the "matrix" $\boldsymbol{c}$

$$
(\mathbf{1}+\mathbf{R})=\left[\begin{array}{lll}
c_{11} & c_{12} & c_{13} \\
c_{21} & c_{22} & c_{23} \\
c_{31} & c_{32} & c_{33}
\end{array}\right]
$$

Then we have

$$
\overline{\mathrm{f}}^{n}=\overline{\mathrm{f}}^{m(\circ)} c_{m n}
$$

Explicitly

$$
\begin{aligned}
& \overline{\mathrm{f}}^{1}=\overline{\mathrm{f}}^{1(\circ)} c_{11}+\overline{\mathrm{f}}^{2(\circ)} c_{21}+\overline{\mathrm{f}}^{3(\circ)} c_{31} \\
& \overline{\mathrm{f}}^{2}=\overline{\mathrm{f}}^{1(\circ)} c_{12}+\overline{\mathrm{f}}^{2(\circ)} c_{22}+\overline{\mathrm{f}}^{3(\circ)} c_{32} \\
& \overline{\mathrm{f}}^{3}=\overline{\mathrm{f}}^{1(\circ)} c_{13}+\overline{\mathrm{f}}^{2(\circ)} c_{23}+\overline{\mathrm{f}}^{3(\circ)} c_{33}
\end{aligned}
$$

We can see that the previous equations are the co mponents of equation (1) (after multiplication) when we choose the case of three emitters and three receptors. So that the three relations can be written as

$$
\left(\bar{f}^{-1}, \bar{f}^{-2}, \mathrm{f}^{-3}\right)=\left(\bar{f}^{-1(0)}, \mathrm{f}^{2(\circ)}, \mathrm{f}^{-3(\circ)}\right)\left[\begin{array}{lll}
c_{11} & c_{12} & c_{13} \\
c_{21} & c_{22} & c_{23} \\
c_{31} & c_{32} & c_{33}
\end{array}\right]
$$

By substituting the expressions for the "matrix" $\mathrm{c}$ then

$$
\begin{aligned}
& \left(\bar{f}^{-1}, \bar{f}^{-2}, \bar{f}^{-3}\right)=
\end{aligned}
$$

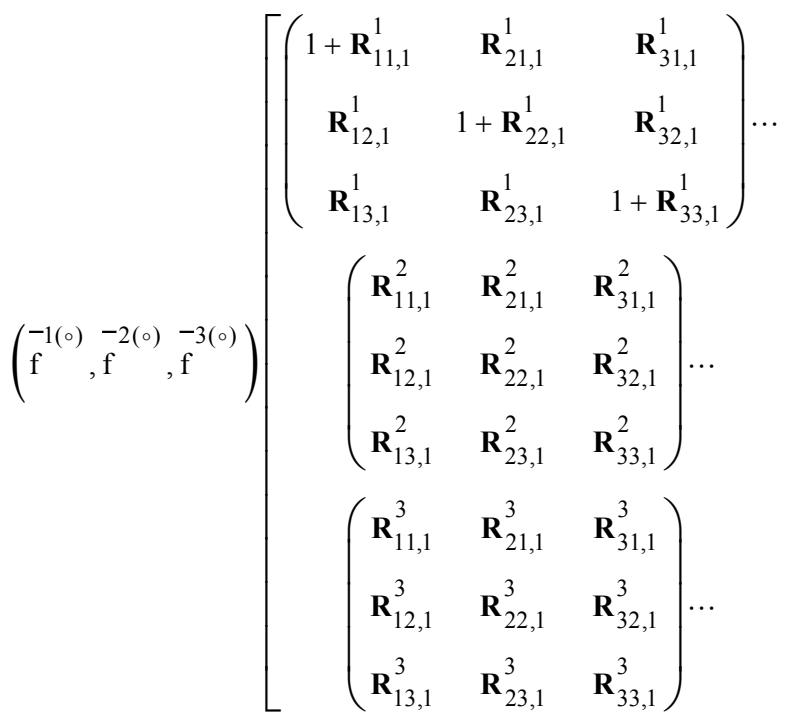

$$
\begin{aligned}
& \cdots\left(\begin{array}{lll}
\mathbf{R}_{11,2}^{1} & \mathbf{R}_{21,2}^{1} & \mathbf{R}_{31,2}^{1} \\
\mathbf{R}_{12,2}^{1} & \mathbf{R}_{22,2}^{1} & \mathbf{R}_{32,2}^{1} \\
\mathbf{R}_{13,2}^{1} & \mathbf{R}_{23,2}^{1} & \mathbf{R}_{33,2}^{1}
\end{array}\right) \quad\left(\begin{array}{lll}
\mathbf{R}_{11,3}^{1} & \mathbf{R}_{21,3}^{1} & \mathbf{R}_{31,3}^{1} \\
\mathbf{R}_{12,3}^{1} & \mathbf{R}_{22,3}^{1} & \mathbf{R}_{32,3}^{1} \\
\mathbf{R}_{13,3}^{1} & \mathbf{R}_{23,3}^{1} & \mathbf{R}_{33,3}^{1}
\end{array}\right) \\
& \cdots\left(\begin{array}{ccc}
1+\mathbf{R}_{11,2}^{2} & \mathbf{R}_{21,2}^{2} & \mathbf{R}_{31,2}^{2} \\
\mathbf{R}_{12,2}^{2} & 1+\mathbf{R}_{22,2}^{2} & \mathbf{R}_{32,2}^{2} \\
\mathbf{R}_{13,2}^{2} & \mathbf{R}_{23,2}^{2} & 1+\mathbf{R}_{33,2}^{2}
\end{array}\right)\left(\begin{array}{lll}
\mathbf{R}_{11,3}^{2} & \mathbf{R}_{21,3}^{2} & \mathbf{R}_{31,3}^{2} \\
\mathbf{R}_{12,3}^{2} & \mathbf{R}_{22,3}^{2} & \mathbf{R}_{32,3}^{2} \\
\mathbf{R}_{13,3}^{2} & \mathbf{R}_{23,3}^{2} & \mathbf{R}_{33,3}^{2}
\end{array}\right) \\
& \left.\ldots\left(\begin{array}{lll}
\mathbf{R}_{11,2}^{3} & \mathbf{R}_{21,2}^{3} & \mathbf{R}_{31,2}^{3} \\
\mathbf{R}_{12,2}^{3} & \mathbf{R}_{22,2}^{3} & \mathbf{R}_{32,2}^{3} \\
\mathbf{R}_{13,2}^{3} & \mathbf{R}_{23,2}^{3} & \mathbf{R}_{33,2}^{3}
\end{array}\right) \quad\left(\begin{array}{ccc}
1+\mathbf{R}_{11,3}^{3} & \mathbf{R}_{21,3}^{3} & \mathbf{R}_{31,3}^{3} \\
\mathbf{R}_{12,3}^{3} & 1+\mathbf{R}_{22,3}^{3} & \mathbf{R}_{32,3}^{3} \\
\mathbf{R}_{13,3}^{3} & \mathbf{R}_{23,3}^{3} & 1+\mathbf{R}_{33,3}^{3}
\end{array}\right)\right)
\end{aligned}
$$

Equations (1), (2), and (3), resume the efforts to make a better envoy of information in a discrete electromagnetic system (for example the broadcast by an antenna arrangement in MIMO technology[11, 12, and 13]) by using time reversal. This equation contains all the necessary information for performing the image envoy (Experimentally a resolution of $\lambda / 30$ is observed on reference[14]).

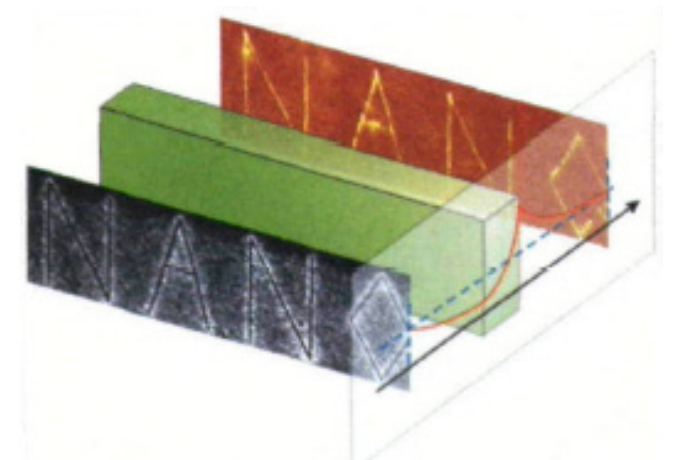

Figure 2. Example of an image with acceptable resolution[3]

\section{Suggested Nanotech Applications}

On reference[15] we showed how we can improve the so called MIMO technology (Multiple Input-Multiple Output); this technology for communications could be scaled to reach Nano-devices in medical applications. But we guess that we can use localized microwave beams to play the role of electromagnetic pincers in the same way as the current optical pincers described in some experiments[4] in which the beam interacts with the spin of the Nano-particles. The possibility to manipulate mic rowaves could make an easiest procedure this extremely difficult issue. Due to the fact that micro waves can interact directly with some molecules and even atoms, maybe we can operate over elaborated Nano-engines and other important Nano-structures. In figure (3) we present the results of a real experiment realized by K. Volke et al. [4] in which they compare the effect of two distinct in shape laser beams over a silica sphere immersed in water and it is possible to move it. This is an example of electro magnetic pincers based on laser beams but that shows the way to the employ of general electromagnetic waves including mic rowaves not necessary in the form of a "maser" beam (that is the precursor of the laser). Then one of our 
strongest proposals is to manipulate Nano-structures with the aid of microwaves controlled by the TR tools including the vector-matrix formalis $\mathrm{m}$.

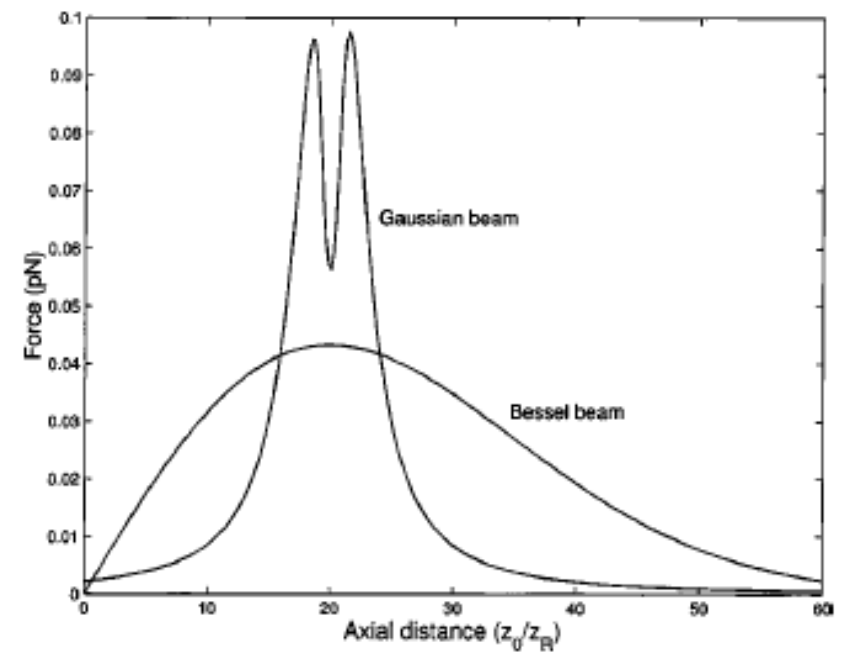

Figure 3. K. Volke et. al experiment [2]. Comparison of the axial optical forces exerted on silica sphere immersed in water $(n=1.087)$ by a Bessel beam and by a Gaussian beam. The parameters for the beams are the following: the radius of the central bright spot of the Bessel beam is $\rho_{B}=0.9 \omega_{0}$, its maximum propagation distance $z_{\max }=40 z_{R}$, and its total power $P=4 P_{0}$, where $\omega_{0}$ is the waist spot size of the Gaussian beam, $Z_{R}$ is its Rayleigh range in the medium, and $P_{0}=1 \mathrm{~mW}$ is its total power. The radius of the sphere is $R=1.5 \omega_{0}$. These curves are exactly the same regardless of the specific value of $\omega_{0}$ and the wavelengths of the laser light provided the ratios between the parameters of both beams and with the size of the particle are preserved

\section{Conclusions}

Even we cannot show yet an application to nanotechnology this paper can shows theoretical results that are original and that can be the mathematical base to real experiments on communications [15], also in this place we show how we can display the forma lis $m$ in the case of three emitters and three receptors, so we can see that the introduced notation is very compact an appropriate for easy use in many branches including Nano-technology. The proposal has been supported by the reference of real manipulation of Nano-structures with electromagnetic beams (laser beams).

If we want to apply our results to design a specific device involving magnetic moment interactions we must built the kernel (3) which defines how the target (for example silica spheres) reacts with the electromagnetic beam, but now we can also focus it in a subwavelength manner. In the other hand, we can reach targets with a very minor size (thirty times minor) with this new technology.

\section{REFERENCES}

[1] D. I. Schuster, A. A. Houck, J. A. Shreier, A. Wallraff, J. M. Gambetta, A. Blais, L. Frunzio, B. Johnson, M. H. Devoret, S. M. Girvin, R. J. Schoelkopf, Resolving photon number states in a superconducting circuit, Nature (London) 445, 515-518 (2007).

[2] V. Jovanov, T. Eissfeller, S. Kapfinger, E. C. Clark, F. Klotz, M. Bichler, J. G. Keizer, P. M. Koenraad, M. S. Brandt, G. Abstreiter, and J. J. Finley, Highly nonlinear excitonic Zeeman spin splitting in composition-engineered artificial atoms, Phys. Rev. B 85, 165433 (2012).

[3] Araneo, R., Lovat, G., Burghignoli, P. and Falconi, C. (2012), Piezo-Semiconductive Quasi-1D Nanodevices with or without Anty-Symetry. Adv. Mater.. doi: 10.1002/adma.201104588.

[4] K. Volke-Sepulveda, S. Chávez-Cerda, V. Garcés-Chávez and K. Dholaskia, Three-dimensional optical forces and transfer of orbital angular momentum from multiringed light beams to spherical microparticles, J. Opt. Soc. Am. B, Vol. 21, No. 10, 1749-1757, October 2004.

[5] K. Volke-Sepulveda, Angular Momentum in Optics and Acoustics: complementary studies, Laser Science, OSA Technical Digest (Optical Society of America, 2011), paper LThC2.

http://www.opticsinfobase.org/abstract.efm?URI=LS-2011LThC2

[6] A. V. Arzola, K. Volke-Sepúlveda, and J.L. Mateos, Experimental Control of Transport and Current Reversals in a Deterministic Optical Rocking Ratchet, arXiv:1104.5171v1[cond-mat.soft] 27 Apr 2011.

[7] David Smith, Superlens breaks optical barrier, Physics World, Institute of Physics Publishing, (2006).

[8] M. Kadic, T. Buckmann, N. Stenger, M. Thiel, and M. Wegener, On the practicability of pentamode mechanical metamaterials, Appl. Phys. Lett. 100, 191901 (2012); doi: 10.1063/1.4709436. http://dx.doi.org/10.1063/1.4709436

[9] J. M. Velázquez-Arcos, J. Granados-Samaniego, J. L. Fernández- Chapou and A. L. Rodríguez-Soria. The Equivalence Between Time Reversed Means and Employment of Left Hand Materials to Overcome the Diffraction Limit. PIERS Proceedings (ISSN 1559-9450), Vol. PIERS 2009 in Moscow Proceedings (ISBN: 978-1-934142-10-3, doi: 10.2529/PIER SOnline), 520-528, published by The Electromagnetics Academy, Cambridge, MA.USA.

[10] Velázquez-Arcos, J. M.; Granados-Samaniego, J.; Chapou, J. L. Fernandez; Vargas, C. A.; , Vector generalization of the discrete Time Reversal formalism brings an electromagnetic application on overcoming the diffraction limit, Electromagnetics in Advanced Applications (ICEAA), 2010 International Conference on , vol., no., pp.264-267, 20-24 Sept. 2010 doi: 10.1109/ICEAA.2010.5653059.

[11] Han, M. and Choi, J. (2012), Compact multiband mimo antenna for next generation USB dongle applications. Microw. Opt. Technol. Lett., 54: 246-250. doi: 10.1002/mop.26478

[12] Yunawei Jin and José M.F. Moura, Time-Reversal Detection Using Antenna Arrays, IEEE TRANSACTIONS ON 
SIGNAL PROCESSING, VOL. 57, NO. 4, APRIL, 2009.

[13] S. Sanayei and A. Nosratinia, Antenna Selection in MIMO Systems, Communications Magazine, IEEE, Vol. 42, No. 10, pp. 68-73 October, 2004.

[14] G. Lerosey, J. de Rosny, A. Tourin, M. Fink. Focusing Beyond the Diffraction Limit with Far-Field Time Reversal,
Science 315, pp. 1120-1122, 2007.

[15] J. M. Velázquez-Arcos, J. Granados-Samaniego, J. L. Fernández-Chapou, C. A. Vargas, Resonances on Discrete Electromagnetic Time Reversal Applications. Electromagnetics in Advanced Applications (ICEAA), 2011 International Conference pp.167-170, 12-16 Sept. 2011 doi: 10.1109/ICEAA.2011.6046297. 